Pacific Journal of Mathematics

AN ORDERING FOR THE BANACH SPACES 


\title{
AN ORDERING FOR THE BANACH SPACES
}

\author{
G. A. EDGAR
}

A binary relation will be defined on the class of all Banach spaces. The relation is transitive and symmetric, so it is natural to call it an "ordering". (The definition also makes sense for locally convex spaces with good duality properties, but this will not be pursued here.) Many of the elementary properties of the ordering are spelled out. Although some connections with Pettis integration and unique preduals have been found, the usefulness of this ordering in Banach space theory remains to be determined.

Notation and terminology used in this paper generally matches Dunford and Schwartz [4], Chapters IV and VI. More recent results in Banach space theory will usually be quoted from Lindenstraus and Tzafriri [11] or from Diestel and Uhl [3]. If $\mathfrak{X}$ is a Banach space, its dual will be denoted $\mathfrak{X}^{*}$, its bidual $\mathfrak{X}^{* *}$. The subset of $\mathfrak{X}^{* *}$ canonically identified with $\mathfrak{X}$ will simply be written $\mathfrak{X}$.

Definition. Let $\mathfrak{X}$ and $\mathfrak{Y}$ be Banach spaces. Then $\mathfrak{X} \prec \mathfrak{Y}$ means

$$
\mathfrak{X}=\cap T^{* *-1}[\mathfrak{Y}],
$$

where the intersection is over all bounded linear operators $T: \mathfrak{X} \rightarrow \mathfrak{V}$.

The definition can be rephrased as follows: $\mathfrak{X} \prec \mathfrak{Y}$ if and only if any $\alpha \in \mathfrak{X}^{* *}$, such that $T^{* *}(\alpha) \in \mathfrak{Y}$ for all bounded linear operators $T$ : $\mathfrak{X} \rightarrow \mathfrak{V}$, must be in $\mathfrak{X}$.

A single operator $T$ with $\mathfrak{X}=T^{* *-1}[\mathfrak{Y}]$ has been called a Tauberian operator (see [10]). If there exists a Tauberian operator $\mathfrak{X} \rightarrow \mathfrak{Y}$, then $\mathfrak{X} \prec \mathfrak{V}$, but not conversely.

Following [9], where the case $\mathfrak{V}=l_{1}$ is considered, we define the $\mathfrak{Y}$-frame (cadre) of $\mathfrak{X}$ by

$$
\mathfrak{F}(\mathfrak{X}, \mathfrak{Y})=\cap T^{* *-1}[\mathfrak{Y}],
$$

with intersection over all operators $T: \mathfrak{X} \rightarrow \mathfrak{Y}$. Then $\mathfrak{F}(\mathfrak{X}, \mathfrak{Y})$ is a Banach space, $\mathfrak{X} \subseteq \mathfrak{F}(\mathfrak{X}, \mathfrak{Y}) \subseteq \mathfrak{X}^{* *}$. One extreme possibility is $\mathfrak{F}(\mathfrak{X}, \mathfrak{V})=\mathfrak{X}^{* *}$, 
which means that every operator from $\mathfrak{X}$ to $\mathfrak{Y}$ is weakly compact. The other extreme is $\mathfrak{F}(\mathfrak{X}, \mathfrak{Y})=\mathfrak{X}$, which is the relation $\mathfrak{X} \prec \mathfrak{V}$ considered here.

Many properties of the relation $\prec$ are stated below. When they are stated without proof, they can be verified by straightforward diagram chasing from the definition.

The relation is transitive (if $\mathfrak{X}_{1} \prec \mathfrak{X}_{2}$ and $\mathfrak{X}_{2} \prec \mathfrak{X}_{3}$, then $\mathfrak{X}_{1} \prec \mathfrak{X}_{3}$ ) and reflexive $(\mathfrak{X} \prec \mathfrak{X}$ for any Banach space $\mathfrak{X})$. So it defines a partial order on the equivalence classes defined by the equivalence relation $\mathfrak{X} \sim \mathfrak{Y}$ iff $\mathfrak{X} \prec \mathfrak{Y}$ and $\mathfrak{Y} \prec \mathfrak{X}$. This partial order is not a total order (see remarks following Proposition 11).

If $\mathfrak{X}$ is isomorphic to a closed subspace of $\mathfrak{Y}$ (below I will say “ $\mathfrak{X}$ embeds in $\mathfrak{Y}$ "), then $\mathfrak{X} \prec \mathfrak{Y}$. This can be seen using the isomorphic embedding $\mathfrak{X} \rightarrow \mathfrak{Y}$ in place of $T$ in the definition. The converse is false, however. We have $l_{3} \prec l_{2}$ (Proposition 1) and $l_{1} \prec c_{0}$ (Proposition 2), but $l_{3}$ does not embed in $l_{2}$ and $l_{1}$ does not embed in $c_{0}$.

The relation $\prec$ has no relation with "semi-embedding", defined by Rosenthal [12]. The space $l_{1}$ semi-embeds in $l_{2}$, but $l_{1} \nless l_{2}$ (Proposition 1); conversely, $L_{1} \prec l_{1}$ (Proposition 10), but $L_{1}$ does not semi-embed in $l_{1}$.

There is a least equivalence class, namely the class consisting of all reflexive spaces. That is:

1. Proposition. If $\mathfrak{X}$ is reflexive, then $\mathfrak{X} \prec \mathfrak{V}$ for all Banach spaces $\mathfrak{Y}$. If $\mathfrak{Y}$ is reflexive, then $\mathfrak{X} \prec \mathfrak{Y}$ if and only if $\mathfrak{X}$ is reflexive.

Proof. Suppose $\mathfrak{X}$ is reflexive. Since

$$
\mathfrak{X} \subseteq \cap T^{* *-1}[\mathfrak{Y}] \subseteq \mathfrak{X}^{* *},
$$

we have equality. Now suppose $\mathfrak{Y}$ is reflexive. Then

$$
\cap T^{* *-1}[\mathfrak{Y}]=\mathfrak{X}^{* *},
$$

so $\mathfrak{X} \prec \mathfrak{Y}$ implies $\mathfrak{X}=\mathfrak{X}^{* *}$.

There is a second-to-least equivalence class, namely the class containing $l_{1}$. That is:

2. Proposition. Let $\mathfrak{Y}$ be a Banach space. Then $l_{1} \prec \mathfrak{V}$ if and only if $\mathfrak{Y}$ is not reflexive. 
Proof. If $l_{1} \prec \mathfrak{V}$, then $\mathfrak{Y}$ is not reflexive by Proposition 1 .

Conversely, suppose $\mathfrak{Y}$ is not reflexive. I must show $l_{1} \prec \mathfrak{V}$. If $l_{1}$ embeds in $\mathfrak{Y}$, then clearly $l_{1} \prec \mathfrak{V}$. So we may assume $l_{1}$ does not embed in $\mathfrak{V}$. Now since $\mathfrak{Y}$ is not reflexive, there is a bounded sequence, no subsequence of which converges in the weak topology [4, Theorems V.4.7 and V.6.1]. Then by a theorem of Rosenthal [11, Theorem 2.e.5] there is a subsequence $\left(y_{n}\right)$ which is either equivalent to the unit vector basis of $l_{1}$ or a weak Cauchy sequence. But $l_{1}$ does not embed in $Y$, so $\left(y_{n}\right)$ is a weak Cauchy sequence. Then $\alpha(f)=\lim _{n} f\left(y_{n}\right), f \in \mathfrak{Y}^{*}$, defines $\alpha \in \mathfrak{Y}^{* *}$ and $\alpha \notin \mathfrak{V}$.

Now consider $\mu \in l_{1}^{* *}$ such that $T^{* *}(\mu) \in \mathfrak{Y}$ for all operators $T$ : $l_{1} \rightarrow \mathfrak{Y}$. We must show that $\mu \in l_{1}$. Now $l_{1}^{* *}$ is canonically identified with the space $b a(\mathbf{N}, \mathscr{P}(\mathbf{N}))$ of bounded, finitely additive set functions on $\mathbf{N}[4$, Theorem IV.8.16]. Any such set function $\mu$ can be written as a sum of a purely finitely additive set function (vanishing on all finite sets) and an element of $l_{1}$ itself $\left[3, \mathrm{p}\right.$. 30]. So to show $\mu \in l_{1}$, we may assume $\mu$ is purely finitely additive and show $\mu=0$.

So assume $\mu \in l_{1}^{* *}$ is a purely finitely additive set function on $\mathbf{N}$, and $T^{* *}(\mu) \in \mathfrak{Y}$ for all $T$. Consider an infinite set $A \subseteq \mathbf{N}$. Define $T: l_{1} \rightarrow \mathfrak{Y}$ by: $T\left(e_{n}\right)=y_{n}$ if $n \in A, T\left(e_{n}\right)=0$ if $n \notin A$. (Here $e_{n}$ is the vector $(0,0, \ldots, 0,1,0,0, \ldots)$ with 1 in the $n$th place.) To compute $T^{* *}(\mu)$, let $f \in \mathfrak{Y}^{*}$ and $\varepsilon>0$. Choose $N$ large enough that $\left|f\left(y_{n}\right)-\alpha(f)\right| \leq \varepsilon$ for all $n \geq N$. Then $\left|f\left(T\left(e_{n}\right)\right)-\alpha(f) \chi_{A}(n)\right| \leq \varepsilon$ except for finitely many $n$. But $\mu$ vanishes on finite sets, so $\left|T^{* *}(\mu)(f)-\alpha(f) \mu(A)\right| \leq \varepsilon$. Thus $T^{* *}(\mu)$ $=\mu(A) \alpha$. But $T^{* *}(\mu) \in \mathfrak{V}$ and $\alpha \notin \mathfrak{V}$, so $\mu(A)=0$. The set $A$ was arbitrary so $\mu=0$. This shows $l_{1} \prec \mathfrak{V}$.

More information on the class of $l_{1}$ is given below (Propositions 10 and 13).

A Banach space $X$ satisfies the condition of Mazur iff any $\alpha \in \mathfrak{X}^{* *}$ which is sequentially continuous on $\left(\mathfrak{X}^{*}\right.$, weak $\left.{ }^{*}\right)$ is actually continuous there (and hence is an element of $\mathfrak{X}$ ).

3. Proposition. A Banach space $\mathfrak{X}$ satisfies the condition of Mazur if and only if $\mathfrak{X} \prec c_{0}$. 
Proof. Suppose $\mathfrak{X} \prec c_{0}$. Let $\alpha \in \mathfrak{X}^{* *}$, and suppose $\alpha$ is sequentially continuous on $\left(\mathfrak{X}^{*}\right.$, weak*). Let $T: \mathfrak{X} \rightarrow c_{0}$ be any operator. Define $f_{n} \in \mathfrak{X}^{*}$ by $f_{n}(x)=T(x)(n)$. Now $\lim _{n} f_{n}(x)=0$ for all $x$, so $f_{n} \rightarrow 0$ (weak*). By hypothesis, $\alpha\left(f_{n}\right) \rightarrow 0$. But $T^{* *}(\alpha) \in l_{\infty}$ is given by $T^{* *}(\alpha)(n)=\alpha\left(f_{n}\right)$. Thus $T^{* *}(\alpha) \in c_{0}$. This holds for all $T: \mathfrak{X} \rightarrow c_{0}$. Therefore $\alpha \in \mathfrak{X}$. So $\mathfrak{X}$ satisfies the condition of Mazur.

Conversely, suppose $\mathfrak{X}$ satisfies the condition of Mazur. Let $\alpha \in$ $\mathfrak{F}\left(\mathfrak{X}, c_{0}\right)$. I claim $\alpha$ is sequentially continuous on $\left(\mathfrak{X}^{*}\right.$, weak $\left.{ }^{*}\right)$. Indeed, let $f_{n} \rightarrow f\left(\right.$ weak $\left.^{*}\right)$ in $\mathfrak{X}^{*}$. Then the operator $T: \mathfrak{X} \rightarrow c_{0}$ defined by $T(x)(n)=$ $\left(f_{n}-f\right)(x)$ has $T^{* *}(\alpha) \in l_{\infty}$ given by $T^{* *}(\alpha)(n)=\alpha\left(f_{n}-f\right)$. But $\alpha \in$ $T^{* *-1}\left[c_{0}\right]$, so $\alpha\left(f_{n}\right) \rightarrow \alpha(f)$. Thus $\alpha$ is weak* sequentially continuous. Thus $\alpha \in X$. This proves $\mathfrak{X} \prec c_{0}$.

There is a largest class containing separable Banach spaces. Namely:

4. Corollary. If $\mathfrak{X}$ is separable, then $\mathfrak{X} \prec c_{0}$.

Proof. Suppose $\mathfrak{X}$ is separable, and $\alpha \in \mathfrak{X}^{* *}$ is sequentially continuous on $\left(\mathfrak{X}^{*}\right.$, weak*). Then the unit hall $B_{\mathfrak{X}^{*}}$ of $\mathfrak{X}^{*}$ has metrizable weak* topology [4, Theorem V.5.1], so $\alpha$ is continuous on $\left(B_{\mathfrak{X}^{*}}\right.$, weak $\left.{ }^{*}\right)$, and therefore [4, Theorem V.5.6] $\alpha \in \mathfrak{X}$. So $\mathfrak{X} \prec c_{0}$ by Proposition 3.

5. Proposition. Let $\mathfrak{Y}$ be a Banach space. Then $c_{0} \prec \mathfrak{V}$ if and only if $c_{0}$ embeds in $\mathfrak{Y}$.

Proof. If $c_{0}$ embeds in $\mathfrak{Y}$, then clearly $c_{0} \prec \mathfrak{V}$. Conversely, suppose $c_{0} \prec \mathfrak{V}$. Then there is an operator $T: c_{0} \rightarrow \mathfrak{V}$ that is not weakly compact. But then $T$ is an isomorphism on some subspace of $c_{0}$ isomorphic to $c_{0}[3$, Theorem 15, p. 159]. Thus $c_{0}$ embeds in $\mathfrak{Y}$.

If $S$ is an infinite compact metric space, then $C(S) \sim c_{0}$. The reader may find it interesting to write down exactly what $\mathfrak{X} \prec C(S)$ means, using [4, Theorem VI.7.1]. Then observe that the result is equivalent to the condition of Mazur (by Proposition 3).

The condition (b) described in the next result appeared first in [6, Proposition 4.4] in connection with the Pettis integral. 
6. Proposition. Let $\mathfrak{X}$ be a Banach space. Then the following are equivalent.

(a) $\mathfrak{X} \prec l_{\infty}$.

(b) If $\alpha \in \mathfrak{X}^{* *}$ is weak ${ }^{*}$ continuous on all bounded weak*-separable subsets of $\mathfrak{X}^{*}$, then $\alpha \in \mathfrak{X}$.

Proof. Suppose $\mathfrak{X} \prec l_{\infty}$. Let $\alpha \in \mathfrak{X}^{* *}$, and suppose $\alpha$ is continuous on all bounded separable subsets $A$ of $\left(\mathfrak{X}^{*}\right.$, weak*). Let $T: \mathfrak{X} \rightarrow l_{\infty}$ be any operator. Then (since the ball of $l_{\infty}^{*}$ is separable), $A=T^{*}\left(B_{l_{\infty}^{*}}\right)$ is a bounded weak*-separable set. By hypothesis, $\alpha$ is weak*-continuous on $A$. Then $T^{* *}(\alpha)=\alpha \circ T^{*}$ is weak* continuous on $B_{l_{\infty}^{*}}$, so $T^{* *}(\alpha) \in l_{\infty}$. Thus $\alpha \in T^{* *-1}\left[l_{\infty}\right]$. But $T$ was any operator, so $\alpha \in \mathfrak{F}\left(\mathfrak{X}, l_{\infty}\right)$. Since $\mathfrak{X} \prec l_{\infty}$, we have $\alpha \in \mathfrak{X}$.

Conversely, assume (b). Let $\alpha \in \mathfrak{F}\left(\mathfrak{X}, l_{\infty}\right)$. Let $A$ be a bounded,

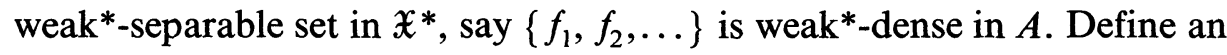
operator $T: \mathfrak{X} \rightarrow l_{\infty}$ by $T(x)(n)=f_{n}(x)$. Then by hypothesis $\alpha \in$ $T^{* *-1}\left[l_{\infty}\right]$, i.e., $T^{* *}(\alpha) \in l_{\infty}$, or $\alpha \circ T^{*}$ is weak ${ }^{*}$-continuous on $B_{l_{\infty}^{*}}$. But then I claim that $\alpha$ is weak ${ }^{*}$-continuous on $T^{*}\left(B_{l_{\infty}^{*}}\right)$. Indeed, suppose $\left(g_{\xi}\right)$ is a net in $T^{*}\left(B_{l_{\infty}^{*}}\right)$ and $g_{\xi} \rightarrow g$. Choose $g_{\xi}^{\prime} \in B_{l_{\infty}^{*}}$ with $T^{*}\left(g_{\xi}^{\prime}\right)=g_{\xi}$. There is a subnet $g_{\xi^{\prime}}^{\prime}$ such that $g_{\xi^{\prime}}^{\prime}$ converges, say to $g^{\prime} \in B_{l_{\infty}^{*}}$. Then $T^{*}\left(g^{\prime}\right)=$ $\lim T^{*}\left(g_{\xi^{\prime}}^{\prime}\right)=\lim g_{\xi^{\prime}}=g$. Thus, $\alpha\left(g_{\xi^{\prime}}\right)=\alpha\left(T^{*}\left(g_{\xi^{\prime}}^{\prime}\right)\right) \rightarrow \alpha\left(T^{*}\left(g^{\prime}\right)\right)=\alpha(g)$. So $\alpha$ is weak*-continuous on $T^{*}\left(B_{l_{\infty}^{*}}\right) \supseteq A$. So $\alpha \in \mathfrak{X}$.

If the word "bounded" is omitted in condition (b), the resulting condition characterizes Banach spaces whose weak topology is real compact [2, Lemma 9]. Talagrand [13] has shown that the two conditions (with and without the word "bounded") are not equivalent.

7. Proposition. Let $\mathfrak{Y}$ be a Banach space. Then $l_{\infty} \prec \mathfrak{Y}$ if and only if $l_{\infty}$ embeds in $\mathfrak{Y}$.

Proof. If $l_{\infty}$ embeds in $\mathfrak{Y}$, then clearly $l_{\infty} \prec \mathfrak{Y}$. Conversely, suppose $l_{\infty} \prec \mathfrak{V}$. Then there is an operator $T: l_{\infty} \rightarrow \mathfrak{V}$ that is not weakly compact. But then $T$ is an isomorphism on a subspace of $l_{\infty}$ isomorphic to $l_{\infty}[3$, Theorem 10, p. 156]. Thus $l_{\infty}$ embeds in $\mathfrak{Y}$.

My original interest in this relation can be traced to the following proposition. Background for this result can be found in [5], [6]. 
8. Proposition. Let $\mathfrak{X}, \mathfrak{Y}$ be Banach spaces and let $(\Omega, \mathscr{F}, \mu)$ be a finite measure space. If $\mathfrak{Y}$ has the $\mu$-Pettis integral property and $\mathfrak{X} \prec \mathfrak{Y}$, then $\mathfrak{X}$ also has the $\mu-P I P$.

Proof. Let $\varphi: \Omega \rightarrow \mathfrak{X}$ be a bounded scalarly measurable function, and let $A \in \mathscr{F}$. Define $\alpha \in X^{* *}$ by $\alpha(f)=\int_{A} f \circ \varphi d \mu, f \in \mathfrak{X}^{*}$. I must show that $\alpha \in \mathfrak{X}$. This will be done by showing that $\alpha \in \mathfrak{F}(\mathfrak{X}, \mathfrak{Y})$. Let $T$ : $\mathfrak{X} \rightarrow \mathfrak{Y}$ be an operator. Then $T \circ \varphi$ is bounded and scalarly measurable $\Omega \rightarrow \mathfrak{Y}$. By hypothesis, the Pettis integral $y=\int_{A} T \circ \varphi d \mu$ exists in $\mathfrak{Y}$. Now for $g \in \mathfrak{Y}^{*}$, we have

$$
\begin{aligned}
T^{* *}(\alpha)(g) & =\alpha\left(T^{*}(g)\right)=\int_{A} T^{*}(g)(\varphi(\omega)) \mu(d \omega) \\
& =\int_{A} g(T(\varphi(\omega))) \mu(d \omega)=g(y) .
\end{aligned}
$$

So $T^{* *}(\alpha)=y \in \mathfrak{Y}$. Thus $\alpha \in \mathfrak{F}(\mathfrak{X}, \mathfrak{Y})=\mathfrak{X}$. This shows that the Pettis integral $\int_{A} \varphi d \mu$ exists in $\mathfrak{X}$. Thus $\mathfrak{X}$ has the $\mu$-PIP.

Corollaries of this result include: If $\mathfrak{X}$ satisfies the condition of Mazur, then $\mathfrak{X}$ has the PIP [6]. If $\mathfrak{X} \prec l_{\infty}$ then $\mathfrak{X}$ has the $\mu$-PIP for all $\mu$ such that $l_{\infty}$ has the $\mu$-PIP [6, Proposition 4.4].

9. Proposition. If $\mathfrak{Y}$ is weakly sequentially complete and $\mathfrak{X} \prec \mathfrak{Y}$, then $\mathfrak{X}$ is weakly sequentially complete.

Proof. Let $\left(x_{n}\right) \subseteq \mathfrak{X}$ be a weakly Cauchy sequence. Define $\alpha \in \mathfrak{X}^{* *}$ by $\alpha(f)=\lim f\left(x_{n}\right)$. In order to show $\alpha \in X$, we will show $\alpha \in \mathfrak{F}(\mathfrak{X}, \mathfrak{V})$. Let $T: \mathfrak{X} \rightarrow \mathfrak{Y}$ be an operator. Then $\left(T\left(x_{n}\right)\right)$ is a weakly Cauchy sequence in $\mathfrak{Y}$, so it converges weakly, say to $y \in \mathfrak{V}$. Then for $g \in \mathfrak{Y}^{*}$,

$$
\begin{aligned}
T^{* *}(\alpha)(g) & =\alpha\left(T^{*}(g)\right)=\lim T^{*}(g)\left(x_{n}\right) \\
& =\lim g\left(T\left(x_{n}\right)\right)=g(y) .
\end{aligned}
$$

So $T^{* *}(\alpha)=y \in \mathfrak{Y}$. This shows $\alpha \in \mathfrak{F}(\mathfrak{X}, \mathfrak{Y})=\mathfrak{X}$. So $\left(x_{n}\right)$ converges weakly to $\alpha \in \mathfrak{X}$.

A condition on a separable Banach space $\mathfrak{X}$ stronger than weak sequential completeness is the following: if $\alpha \in \mathfrak{X}^{* *}$ is Borel measurable on $\left(\mathfrak{X}^{*}\right.$, weak*), then $\alpha \in \mathfrak{X}$. An argument like the preceding one shows that if $\mathfrak{X} \prec \mathfrak{V}$ and $\mathfrak{Y}$ satisfies this condition, then so does $\mathfrak{X}$. It seems to be 
unknown whether this condition is equivalent to weak sequential completeness (in a Banach space). Any counterexample would have to be a space that fails $\mathfrak{X} \prec l_{1}$. (See note added in proof.)

Godefroy and Talagrand [9] say that a Banach space $\mathfrak{X}$ has property (X) iff any $\alpha \in \mathfrak{X}^{* *}$ such that $\alpha\left(\sum f_{n}\right)=\sum \alpha\left(f_{n}\right)$, for every sequence $\left(f_{n}\right) \subset \mathfrak{X}^{*}$ with $\Sigma\left|f_{n}(x)\right|<\infty$ for all $x \in \mathfrak{X}$, must be in $\mathfrak{X}$. (The sum $\Sigma f_{n}$ is taken in the weak* topology of $\mathfrak{X}^{*}$.)

10. Proposition. Let $\mathfrak{X}$ be a Banach space. Then $\mathfrak{X} \prec l_{1}$ if and only if $\mathfrak{X}$ has property $(\mathrm{X})$.

Proof. Suppose $\mathfrak{X} \prec l_{1}$. Let $\alpha \in \mathfrak{X}^{* *}$, and suppose $\alpha\left(\sum f_{n}\right)=\sum \alpha\left(f_{n}\right)$ for every sequence $\left(f_{n}\right) \subset \mathfrak{X}^{*}$ with $\Sigma\left|f_{n}(x)\right|<\infty$ for all $x \in \mathfrak{X}$. (This is a "weakly unconditionally Cauchy" series.) To show that $\alpha \in \mathfrak{X}$, we show that $\alpha \in \mathfrak{F}\left(\mathfrak{X}, l_{1}\right)$. Let $T: \mathfrak{X} \rightarrow l_{1}$ be an operator. Write $e_{n}$ for the canonical unit vectors in $l_{1}^{*}=l_{\infty}$. Let $f_{n}=T^{*}\left(e_{n}\right)$. Then for any $x \in \mathfrak{X}$, we have

$$
\sum\left|f_{n}(x)\right|=\sum\left|e_{n}(T(x))\right|=\|T(x)\|<\infty .
$$

Thus $\alpha\left(\sum f_{n}\right)=\sum \alpha\left(f_{n}\right)$. If $\left(a_{n}\right)$ is a bounded sequence of scalars, the same argument shows $\alpha\left(\sum a_{n} f_{n}\right)=\sum a_{n} \alpha\left(f_{n}\right)$. Define $u: \mathbf{N} \rightarrow \mathbf{R}$ by $u(n)=\alpha\left(f_{n}\right)$. Then for any $g=\sum a_{n} e_{n} \in l_{\infty}$, we have

$$
\begin{aligned}
T^{* *}(\alpha)(g) & =\alpha\left(T^{*}(g)\right)=\alpha\left(T^{*}\left(\sum a_{n} e_{n}\right)\right) \\
& =\alpha\left(\sum a_{n} f_{n}\right)=\sum a_{n} \alpha\left(f_{n}\right)=g(u) .
\end{aligned}
$$

So $T^{* *}(\alpha)=u \in l_{1}$. Thus $\alpha \in \mathfrak{F}\left(\mathfrak{X}, l_{1}\right)=\mathfrak{X}$. This shows that property $(\mathrm{X})$ holds.

Conversely, suppose property (X) holds. Let $\alpha \in \mathfrak{X}^{* *}$, and suppose $T^{* *}(\alpha) \in l_{1}$ for all operators $T: \mathfrak{X} \rightarrow l_{1}$. Let $\left(f_{n}\right) \subset \mathfrak{X}^{*}$ with $\Sigma\left|f_{n}(x)\right|<\infty$ for all $x \in \mathfrak{X}$. Then an operator $T: X \rightarrow l_{1}$ is defined by $T(x)(n)=f_{n}(x)$. Since $T^{* *}(\alpha) \in l_{1}$, we have

$$
T^{* *}(\alpha)\left(\sum e_{n}\right)=\sum T^{* *}(\alpha)\left(e_{n}\right),
$$

or

$$
\alpha\left(\sum f_{n}\right)=\sum \alpha\left(f_{n}\right)
$$

So by property (X), we have $\alpha \in \mathfrak{X}$. This shows $\mathfrak{X} \prec l_{1}$. 
Recall that $\mathfrak{X} \prec l_{1}$ implies that either $\mathfrak{X}$ is reflexive or $\mathfrak{X} \sim l_{1}$. Godefroy and Talagrand [9] show that the following Banach spaces have property (X) [assuming that there are no measurable cardinals, or that the spaces are small enough (e.g., separable) that measurable cardinals do not matter].

(i) A subspace of $L_{1}(\mu)$, where $\mu$ is any measure.

(ii) $L_{1} / H_{1}$.

(iii) A weakly sequentially complete direct summand of a Banach lattice.

(iv) Sequentially complete subspace of an order continuous Banach lattice.

(v) Predual of a $W^{*}$-algebra.

(vi) A space with local unconditional structure not containing $l_{\infty}^{n}$ uniformly.

Godefroy and Talagrand studied property (X) in connection with uniqueness of preduals. The ordering $\prec$ can be used in the same way. The following proof can be imitated with many other spaces in place of the James space $J$.

11. TheOREM. Let $J$ be the James quasireflexive space, and let $\mathfrak{X} \prec J$. Then $\mathfrak{X}$ is the unique isometric predual of $\mathfrak{X}^{*}$.

Proof. First note that any predual of $\mathfrak{X}^{*}$ is canonically identified with a subspace of $\mathfrak{X}^{* *}$.

(A) We first prove the following: Let $\mathfrak{X}_{1} \subseteq \mathfrak{X}^{* *}$ be a predual of $\mathfrak{X}^{*}$, and let $S: J^{*} \rightarrow \mathfrak{X}^{*}$ be an operator. Then $S$ is $\sigma\left(J^{*}, J\right)-\sigma\left(\mathfrak{X}^{*}, \mathfrak{X}\right)$ continuous if and only if $S$ is $\sigma\left(J^{*}, J\right)-\sigma\left(\mathfrak{X}^{*}, \mathfrak{X}_{1}\right)$ continuous.

The James space $J$ will be considered to be (as in [7]) the set of all functions $f$ on the ordinal space $[0, \omega]$ satisfying

(i) $f(0)=0$,

(ii) $\lim _{k<\omega} f(k)=f(\omega)$,

(iii) $\|f\|=\sup \left(\sum_{t=1}^{n}\left|f\left(k_{i}\right)-f\left(k_{i-1}\right)\right|^{2}\right)^{1 / 2}<\infty$,

the sup is over all finite sequences $k_{0}<k_{1}<\cdots<k_{n}$ in $[0, \omega]$. The evaluation functionals are defined by $e_{\alpha}(f)=f(\alpha), \alpha \in(0, \omega]$.

So suppose $S$ is $\sigma\left(J^{*}, J\right)-\sigma\left(X^{*}, X\right)$ continuous. Then $S\left(e_{k}\right) \rightarrow S\left(e_{\omega}\right)$ in $\sigma\left(\mathfrak{X}^{*}, \mathfrak{X}\right)$. To show that $S$ is $\sigma\left(J^{*}, J\right)-\sigma\left(\mathfrak{X}^{*}, \mathfrak{X}_{1}\right)$ continuous, it 
suffices to show $S\left(e_{k}\right) \rightarrow S\left(e_{\omega}\right)$ in $\sigma\left(\mathfrak{X}^{*}, \mathfrak{X}_{1}\right)$. Now since $J^{* *}$ can be identified with the set of all $f$ on $[0, \omega]$ satisfying (i) and (iii), above, we know $S\left(e_{k}\right)$ converges in $\sigma\left(\mathfrak{X}^{*}, \mathfrak{X}_{1}\right)$. Write $h$ for the limit of $S\left(e_{k}\right)-S\left(e_{\omega}\right)$ in $\sigma\left(\mathfrak{X}^{*}, \mathfrak{X}_{1}\right)$. I must show $h=0$.

Fix $n$, and let $k_{0}<k_{1}<\cdots<k_{n}$ be positive integers. In $J^{*}$, consider

$$
u=\sum_{i=1}^{n} \frac{(-1)^{n-1}}{\sqrt{n}}\left(e_{k_{t}}-e_{k_{t-1}}\right) \text {. }
$$

Then $\|u\| \leq 1$, so $\|S(u)\| \leq\|S\|$. Thus:

$$
\left\|\sum_{i=1}^{n}(-1)^{n-i} \frac{1}{\sqrt{n}}\left(S\left(e_{k_{i}}-e_{\omega}\right)-S\left(e_{k_{t-1}}-e_{\omega}\right)\right)\right\| \leq\|S\|
$$

or

$$
\begin{gathered}
\frac{1}{\sqrt{n}}\left\|S\left(e_{k_{n}}-e_{\omega}\right)+(-1)^{n+1} S\left(e_{k_{0}}-e_{\omega}\right)+2 \sum_{i=1}^{n-1}(-1)^{n-i} S\left(e_{k_{i}}-e_{\omega}\right)\right\| \\
\leq\|S\| .
\end{gathered}
$$

Now let $k_{n}$ increase; we have $S\left(e_{k_{n}}-e_{\omega}\right) \rightarrow 0$ in $\sigma\left(\mathfrak{X}^{*}, \mathfrak{X}\right)$ as $k_{n} \rightarrow \omega$ and $k_{0}, \ldots, k_{n-1}$ remain fixed, so

$$
\frac{1}{\sqrt{n}}\left\|(-1)^{n+1} S\left(e_{k_{0}}-e_{\omega}\right)+2 \sum_{i=1}^{n-1}(-1)^{n-i} S\left(e_{k_{t}}-e_{\omega}\right)\right\| \leq\|S\| .
$$

Then let $k_{n-1}$ increase; we have $S\left(e_{k_{n-1}}-e_{\omega}\right) \rightarrow h$ in $\sigma\left(\mathfrak{X}^{*}, \mathfrak{X}_{1}\right)$, so

$$
\frac{1}{\sqrt{n}}\left\|(-1)^{n+1} S\left(e_{k_{0}}-e_{\omega}\right)+2 \sum_{i=1}^{n-2}(-1)^{n-i} S\left(e_{k_{l}}-e_{\omega}\right)-2 h\right\| \leq\|S\| .
$$

Then let $k_{n-2}$ increase; we have $S\left(e_{k_{n-2}}-e_{\omega}\right) \rightarrow 0$ in $\sigma(\mathfrak{X}, \mathfrak{X})$. Then let $k_{n-3}$ increase; we have $S\left(e_{k_{n-3}}-e_{\omega}\right) \rightarrow h$ in $\sigma\left(\mathfrak{X}^{*}, \mathfrak{X}_{1}\right)$. We get in the end

$$
\frac{1}{\sqrt{n}}\|n h\| \leq\|S\| \text {. }
$$

But this holds for all $n$, so $h=0$. This shows that $S$ is $\sigma\left(J^{*}, J\right)-\sigma\left(\mathfrak{X}^{*}, \mathfrak{X}_{1}\right)$ continuous.

(B) We next prove the following: if $\mathfrak{X}$ and $\mathfrak{X}_{1}$ have isometric dual spaces, then $\mathfrak{F}(\mathfrak{X}, J)=\mathfrak{F}\left(\mathfrak{X}_{1}, J\right)$. 
We may assume $\mathfrak{X}_{1} \subseteq \mathfrak{X}^{* *}$. Now

$$
\begin{aligned}
\mathfrak{F}(\mathfrak{X}, J) & =\bigcap\left\{T^{* *-1}(J) \mid T: \mathfrak{X} \rightarrow J\right\} \\
& =\bigcap\left\{S^{*-1}(J) \mid S: J^{*} \rightarrow \mathfrak{X}^{*}, S \text { is weak* continuous }\right\}
\end{aligned}
$$

depends only on which operators $S: J^{*} \rightarrow \mathfrak{X}^{*}$ are weak* continuous, and by the above observation this is the same for $\mathfrak{X}_{1}$ and $\mathfrak{X}$.

(C) Finally, suppose $\mathfrak{X} \prec J$ and $\mathfrak{X}_{1}^{*}$ is isometric to $\mathfrak{X}^{*}$. If $\mathfrak{X}_{1}$ is identified with the appropriate subspace of $\mathfrak{X}^{* *}$, we have $\mathfrak{X}_{1} \subseteq \mathfrak{F}\left(\mathfrak{X}_{1}, J\right)$ $=\mathfrak{F}(\mathfrak{X}, J)=\mathfrak{X}$. Similarly $\mathfrak{X} \subseteq \mathfrak{X}_{1}$. So $\mathfrak{X}$ is the unique predual of $\mathfrak{X} *$.

The relation $\prec$ is not a total order on the equivalence classes. The spaces $c_{0}$ and $J\left(\omega_{1}\right)$ of [7] are not comparable. An example using only separable spaces can be obtained using the James quasireflexive space $J$ and Bourgain's $\mathscr{L}_{\infty}$ space with the Schur property $\mathfrak{X}[1]$. Then $J \prec \mathfrak{X}$ fails by Proposition 9 , since $\mathfrak{X}$ is weakly sequentially complete, but $J$ is not. And $\mathfrak{X} \prec J$ fails since $J^{* *}$ contains no copy of $c_{0}$, and $\mathfrak{X}^{* *}$ is isomorphic to an $L_{\infty}(\mu)$ space, so all operators $\mathfrak{X}^{* *} \rightarrow J^{* *}$ are weakly compact [3, VI.1.2], and hence all operators $\mathfrak{X} \rightarrow J$ are weakly compact.

According to Propositions 2, 9, 10, the second-least equivalence class, the class of $l_{1}$, contains all nonreflexive spaces in the list after Proposition 10 , and is contained in the class of all weakly sequentially complete spaces. However the class of $l_{1}$ does not contain all weakly sequentially complete spaces. We next give two illustrations of this.

12. Proposition. Let $\Gamma$ be a set. Then $l_{1}(\Gamma) \prec l_{1}$ if and only if card $\Gamma$ is not a (real-valued) measurable cardinal.

Proof. Recall that $l_{1}(\Gamma)^{*}=l_{\infty}(\Gamma)$ [4, Theorem IV.8.5 and following Remark] and $l_{\infty}(\Gamma)^{*}=b a(\Gamma, \mathcal{P}(\Gamma))$, the space of all finitely additive, bounded, signed measures on the power set $\mathscr{P}(\Gamma)$ of $\Gamma[4$, Theorem IV.8.16]. We claim that the frame $\mathfrak{F}\left(l_{1}(\Gamma), l_{1}\right)$ is the subspace $c a(\Gamma, \mathcal{P}(\Gamma))$ of all countably additive signed measures on $\mathscr{P}(\Gamma)$. Since $l_{1}(\Gamma)=$ $c a(\Gamma, \mathscr{P}(\Gamma))$ if and only if card $\Gamma$ is not measurable, this will prove the result. Note that $l_{1}=c a(\mathbf{N}, \mathcal{P}(\mathbf{N}))$. If $\mu \in b a(\Gamma, \mathscr{P}(\Gamma))$ and $T: l_{1}(\Gamma) \rightarrow l_{1}$, then $T^{* *}(\mu) \in b a(\mathbf{N}, \mathcal{P}(\mathbf{N}))$. If $\mu$ is countably additive, so is $T^{* *}(\mu)$ : If $A_{n} \subseteq \mathrm{N}, A_{n} \downarrow \varnothing$, then $\chi_{A_{n}} \rightarrow 0$ (weak*) in $l_{\infty}$, so $T^{*}\left(\chi_{A_{n}}\right) \rightarrow 0$ (weak*) in $l_{\infty}(\Gamma)$, and thus $T^{*}\left(\chi_{A_{n}}\right) \rightarrow 0$ pointwise, so by the Lebesgue dominated 
convergence theorem, $T^{* *}(\mu)\left(\chi_{A_{n}}\right) \rightarrow 0$. This shows that $\mathfrak{F}\left(l_{1}(\Gamma), l_{1}\right) \supseteq$ $c a(\Gamma, \mathscr{P}(\Gamma))$. Conversely, suppose $\mu \in \mathfrak{F}\left(l_{1}(\Gamma), l_{1}\right)$, and let $B_{n} \subseteq \Gamma, B_{n}$ disjoint. Define $T: l_{1}(\Gamma) \rightarrow l_{1}$ by: $T(f)(n)=\sum_{\gamma \in B_{n}} f(\gamma), f \in l_{1}(\Gamma), n \in \mathbf{N}$. If $e_{n} \in l_{\infty}$ are the usual unit vectors and $u \in l_{\infty}$ is $u(n)=1$, then $\sum e_{n}=u$ (weak*) in $l_{\infty}$. But $T^{* *}(\mu) \in l_{1}=c a(N, \mathscr{P}(\mathbf{N}))$, so $\mu\left(\cup A_{n}\right)=T^{* *}(\mu)(u)$ $=\Sigma T^{* *}(\mu)\left(e_{n}\right)=\Sigma \mu\left(A_{n}\right)$. Thus $\mu \in c a(\Gamma, \mathscr{P}(\Gamma))$. This shows that $\mathfrak{F}\left(l_{1}(\Gamma), l_{1}\right)=c a(\Gamma, \mathcal{P}(\Gamma))$.

Bourgain and Delbaen [1] give an example of a space $\mathfrak{X}$ which is a separable $\mathcal{L}_{\infty}$-space but has the property of Schur (so it is weakly sequentially complete). As noted above, $\mathfrak{X} \nsim l_{1}$. Another reason for this is the following. Since $\mathfrak{X}$ is a $e_{\infty}$-space, so is any complemented subspace, hence no complemented subspace is isomorphic to $l_{1}$. By the next result, $\mathfrak{X}$ is not in the equivalence class of $l_{1}$.

13. THEOREM. Let $\mathfrak{X}$ be a Banach space, and suppose $\mathfrak{X} \prec l_{1}$. Then every bounded sequence in $\mathfrak{X}$ that is not relatively weakly compact has a subsequence equivalent to the unit vector basis of $l_{1}$ with closed span complemented in $\mathfrak{X}$.

Proof. Write $\left(e_{n}\right)_{n=1}^{\infty}$ for the unit vector basis in $l_{1}$, and $\left(e_{n}^{*}\right)_{n=1}^{\infty}$ for the biorthogonal sequence in $l_{\infty}$.

First recall that if $S: \mathfrak{X} \rightarrow l_{1}$ is an operator, and $\left(y_{j}\right)_{j=1}^{\infty}$ is a bounded sequence in $\mathfrak{X}$ with $S\left(y_{j}\right)=e_{j}$ for all $j$, then the span of $\left\{y_{j}\right\}$ is isomorphic to $l_{1}$, since $S$ is an isomorphism there with inverse $R: l_{1} \rightarrow \mathfrak{X}$ defined by $R\left(\sum a_{j} e_{j}\right)=\sum a_{j} y_{j}$, and the span of $\left\{y_{j}\right\}$ is complemented in $\mathfrak{X}$ with projection $P=R S$.

Next recall that if $\left(A_{j}\right)_{j=1}^{\infty}$ are disjoint finite sets in $\mathbf{N}$, then the span of $\left(\chi_{A},\right)_{j=1}^{\infty}$ in $l_{1}$ is a complemented subspace isomorphic to $l_{1}$, since $S$ : $l_{1} \rightarrow l_{1}$ defined by $S\left(\sum_{n=1}^{\infty} a_{n} e_{n}\right)=\sum_{j=1}^{\infty}\left(\sum_{n \in A_{j}} a_{n}\right) e_{j}$ is a map as required above, with $y_{j}=\chi_{A_{j}} /\left\|\chi_{A_{j}}\right\|$.

So suppose $\mathfrak{X} \prec l_{1}$. Let $\left(x_{n}\right)$ be a bounded sequence in $\mathfrak{X}$. Let $\alpha \in \mathfrak{X}^{* *}$ be a fixed cluster point of $\left(x_{n}\right)$. Assume $\alpha \notin \mathfrak{X}$.

Since $\mathfrak{X} \prec l_{1}$, there exists an operator $T: \mathfrak{X} \rightarrow l_{1}$ with $T^{* *}(\alpha) \notin l_{1}$. That is, under the identification $l_{1}^{* *}=b a(\mathbf{N}, \mathscr{P}(\mathbf{N}))$, the measure $A \mapsto$ $\alpha\left(T^{*}\left(\chi_{A}\right)\right)$ is not countably additive on $\mathscr{P}(\mathbf{N})$. So there exist disjoint sets 
$A_{k} \subseteq \mathrm{N}$ with $\sum_{k=1}^{\infty} \alpha\left(T^{*}\left(\chi_{A_{k}}\right)\right) \neq \alpha\left(T^{*}\left(\chi_{\cup A_{k}}\right)\right)$. Composing $T$ with a projection on $l_{1}$, we may assume without loss of generality that $A_{k}=\{k\}$, so that

$$
\sum_{k=1}^{\infty} \alpha\left(T^{*}\left(e_{k}^{*}\right)\right) \neq \alpha\left(T^{*}\left(\chi_{\mathbf{N}}\right)\right) .
$$

In fact (using another projection to suppress a finite number of coordinates) we may assume, for some $\varepsilon>0$, that

$$
\sum_{k=1}^{\infty}\left|\alpha\left(T^{*}\left(e_{k}^{*}\right)\right)\right|<\frac{\varepsilon}{10} \text { and }\left|\alpha\left(T^{*}\left(\chi_{\mathbf{N}}\right)\right)\right|>\varepsilon .
$$

We now construct "blocks" $A_{j}=\left\{a_{j}+1, a_{j}+2, \ldots, a_{j+1}\right\}$ recursively. Let $a_{1}=0$. Choose $m_{1}$ so that

$$
\left|T^{*}\left(\chi_{N}\right)\left(x_{m_{1}}\right)\right|>\varepsilon
$$

(This is possible since $\alpha$ is a cluster point of the sequence $\left(x_{n}\right)$.) Then choose $a_{2}>a_{1}$ so that $A_{1}=\left\{1,2, \ldots, a_{2}\right\}$ satisfies

$$
\left|T^{*}\left(\chi_{A_{1}}\right)\left(x_{m_{1}}\right)\right|>\varepsilon \text { and } \sum_{k \notin A_{1}}\left|T^{*}\left(e_{k}^{*}\right)\left(x_{m_{1}}\right)\right|<\frac{\varepsilon}{10} \text {. }
$$

This specifies $a_{2}, m_{1}$. Next note that

$$
\left|\alpha\left(T^{*}\left(\chi_{\left\{a_{2}+1, a_{2}+2, \ldots\right\}}\right)\right)\right|>\frac{9}{10} \varepsilon .
$$

Choose $m_{2}>m_{1}$ so that

$$
\left|T^{*}\left(\chi_{\left\{a_{2}+1, \ldots\right\}}\right)\left(x_{m_{2}}\right)\right|>\frac{9}{10} \varepsilon \text { and } \sum_{k \in A_{1}}\left|T^{*}\left(e_{k}^{*}\right)\left(x_{m_{2}}\right)\right|<\frac{\varepsilon}{10} .
$$

Then choose $a_{3}>a_{2}$ so that $A_{2}=\left\{a_{2}+1, \ldots, a_{3}\right\}$ satisfies

$$
\left|T^{*}\left(\chi_{A_{2}}\right)\left(x_{m_{2}}\right)\right|>\frac{9}{10} \varepsilon \text { and } \sum_{k=a_{3}+1}^{\infty}\left|T^{*}\left(e_{k}^{*}\right)\left(x_{m_{2}}\right)\right|<\frac{\varepsilon}{10} \text {. }
$$

Continuing in this manner, we get $m_{1}<m_{2}<m_{3}<\cdots$ and $0=a_{1}<a_{2}$ $<a_{3}<\cdots$ so that if $A_{j}=\left\{a_{j}+1, \ldots, a_{j+1}\right\}$, then

$$
\begin{gathered}
\left|T^{*}\left(\chi_{A_{j}}\right)\left(x_{m_{j}}\right)\right|>\frac{9}{10} \varepsilon \text { for all } j, \\
\sum_{k \notin A_{j}}\left|T^{*}\left(e_{k}^{*}\right)\left(x_{m_{j}}\right)\right|<\frac{2}{10} \varepsilon \text { for all } j, \\
\sum_{j=1}^{\infty}\left|\alpha\left(T^{*}\left(\chi_{A_{j}}\right)\right)\right|<\frac{\varepsilon}{10}<\varepsilon<\left|\alpha\left(T^{*}\left(\chi_{\mathbf{N}}\right)\right)\right| .
\end{gathered}
$$


Then (1) and (2) show that $\left(T\left(x_{m}\right)\right)_{j=1}^{\infty}$ spans a complemented subspace of $l_{1}$, and $\left(T\left(x_{m_{j}}\right)\right)$ is equivalent to the unit vector basis of $l_{1}$. Again composing $T$ with a projection on $l_{1}$, we may assume that $T\left(x_{m_{j}}\right)=e_{j}$. [By (3) we still have $T^{* *}(\alpha) \notin l_{1}$.] Thus we finally have that $\left(x_{m_{j}}\right)_{j=1}^{\infty}$ is equivalent to the unit vector basis of $l_{1}$ and spans a complemented subspace of $\mathfrak{X}$.

I do not know whether the converse of this theorem holds. If it does, this is a non-trivial characterization of the equivalence class of $l_{1}$. It can be shown that $\mathfrak{X}<l_{1}$ is equivalent to the following more complicated condition: For any bounded sequence $\left(x_{n}\right)_{n=1}^{\infty}$ in $\mathfrak{X}$ that is not relatively weakly compact, and any cluster point $\alpha$ of $\left(x_{n}\right)$ in $\mathfrak{X}^{* *}$ but not in $\mathfrak{X}$, there exist a subsequence $\left(x_{m_{j}}\right)_{j=1}^{\infty}$ equivalent to the unit vector basis of $l_{1}$ and a projection $T$ onto the closed span of $\left(x_{m_{j}}\right)$ such that $T^{* *}(\alpha) \notin \mathfrak{X}$.

Since there is a least equivalence class (Proposition 1), is there a greatest equivalence class? There is a greatest class containing separable spaces, namely the class of $c_{0}$ (Proposition 3). But there is no greatest class:

14. Proposition. If $\mathfrak{X}$ is any Banach space, then there is a set $\Gamma$ so large that $\mathfrak{X} l_{\infty}(\Gamma)$ but $\mathfrak{X} l_{\infty}(\Gamma)$.

Proof. Let the cofinality of the cardinal of $\Gamma$ exceed the cardinal of $\mathfrak{X}^{*}$. Let $\gamma$ be the least ordinal with the same cardinal as $\Gamma$. We will show that $C([0, \gamma]) \prec \mathfrak{X}$ fails. Since $C([0, \gamma])$ embeds in $l_{\infty}(\Gamma)$, this shows that $l_{\infty}(\Gamma) \prec \mathfrak{X}$ also fails.

To show that $C([0, \gamma]) \prec \mathfrak{X}$ fails, we will exhibit $\alpha \in \mathfrak{F}(C([0, \gamma]), \mathfrak{X})$ with $\alpha \notin C([0, \gamma])$. The dual $C([0, \gamma])^{*}$ can be identified with $l_{1}([0, \gamma])$. Let $\alpha \in l_{1}([0, \gamma])^{*}$ be defined by $\alpha(h)=h(\gamma), h \in l_{1}([0, \gamma])$. Then $\alpha \notin$ $C([0, \gamma])$.

Let $T: C([0, \gamma]) \rightarrow \mathfrak{X}$ be any operator. If $f \in \mathfrak{X}^{*}$, then the function $T^{*}(f) \in l^{1}([0, \gamma])$ vanishes outside some countable subset of $[0, \gamma]$. Since $\gamma$ has cofinality greater than $\operatorname{card}\left(\mathfrak{X}^{*}\right)$, there exists $\gamma_{0}<\gamma$ such that all $T^{*}(f)$ vanish on the interval $\left(\gamma_{0}, \gamma\right)$. Then we have

$$
\begin{aligned}
T^{* *}(\alpha)(f) & =\alpha\left(T^{*}(f)\right)=T^{*}(f)(\gamma) \\
& =T^{*}(f)\left(\chi_{\left(\gamma_{0}, \gamma\right]}\right)=f\left(T\left(\chi_{\left(\gamma_{0}, \gamma\right]}\right)\right)
\end{aligned}
$$


so that $T^{* *}(\alpha)=T\left(\chi_{\left(\gamma_{0}, \gamma\right]}\right) \in \mathfrak{X}$. This shows that $\alpha \in \mathfrak{F}(C([0, \gamma]), \mathfrak{X})$, which completes the proof.

Here is a permanence property of the class of $l_{1}$. Recall that $l_{1}(\Gamma) \prec l_{1}$ is and only if card $\Gamma$ is not measurable.

15. Proposition. Let $\Gamma$ be a set with $l_{1}(\Gamma) \prec l_{1}$. For each $\gamma \in \Gamma$, let $\mathfrak{X}_{\gamma}$ be a Banach space with $\mathfrak{X}_{\gamma} \prec l_{1}$. Then the $l_{1}$-direct sum

$$
\mathfrak{X}=\left(\bigoplus_{\gamma \in \Gamma} \mathfrak{X}_{\gamma}\right)_{1}
$$

satisfies $\mathfrak{X} \prec l_{1}$.

Proof. For each $\gamma \in \Gamma$, let $J_{\gamma}: \mathfrak{X}_{\gamma} \rightarrow \mathfrak{X}$ be the coordinate embedding, and $P_{\gamma}: \mathfrak{X} \rightarrow \mathfrak{X}_{\gamma}$ the coordinate projection. Let $\alpha \in \mathfrak{F}\left(\mathfrak{X}, l_{1}\right)$. For fixed $\gamma$, consider $P_{\gamma}^{* *}(\alpha) \in \mathfrak{X}_{\gamma}^{* *}$. If $S: \mathfrak{X}_{\gamma} \rightarrow l_{1}$ is any operator, then $S P_{\gamma}$ maps $\mathfrak{X}$ to $l_{1}$, so $\left(S P_{\gamma}\right)^{* *}(\alpha) \in l_{1}$. This holds for all $S$, so $P_{\gamma}^{* *}(\alpha) \in \mathfrak{F}\left(\mathfrak{X}_{\gamma}, l_{1}\right)=\mathfrak{X}_{\gamma}$. Write $u_{\gamma}=P_{\gamma}^{* *}(\alpha)$.

Now if $\Gamma_{0} \subseteq \Gamma$ is finite, then

$$
\sum_{\gamma \in \Gamma_{0}}\left\|u_{\gamma}\right\|=\left\|\sum_{\gamma \in \Gamma_{0}} J_{\gamma}\left(u_{\gamma}\right)\right\|=\left\|\sum_{\gamma \in \Gamma_{0}} J_{\gamma} P_{\gamma}^{* *}(\alpha)\right\| \leq\|\alpha\|,
$$

so $u=\sum_{\gamma \in \Gamma} J_{\gamma}\left(u_{\gamma}\right)$ converges in the norm of $\mathfrak{X}$, and $P_{\gamma}(u)=u_{\gamma}$. I claim $u=\alpha$. Let $f \in \mathfrak{X}^{*}$. For each $\gamma \in \Gamma$, let $f_{\gamma}=J_{\gamma}^{*}(f) \in \mathfrak{X}_{\gamma}^{*}$. Define the map $S: \mathfrak{X} \rightarrow l_{1}(\Gamma)$ by $S(x)(\gamma)=f_{\gamma}\left(P_{\gamma}(x)\right)$. Since $\mathfrak{X} \prec l_{1} \prec l_{1}(\Gamma)$, we have $S^{* *}(\alpha) \in l_{1}(\Gamma)$. Now if $\Gamma_{0} \subseteq \Gamma$ is finite, we have

$$
\begin{aligned}
\left(\sum_{\gamma \in \Gamma_{0}} P_{\gamma}^{*}\left(f_{\gamma}\right)\right)(u) & =\sum_{\gamma \in \Gamma_{0}} f_{\gamma}\left(u_{\gamma}\right)=\sum_{\gamma \in \Gamma_{0}} \alpha\left(f_{\gamma}\right) \\
& =\alpha\left(S^{*}\left(\chi_{\Gamma_{0}}\right)\right)=S^{* *}(\alpha)\left(\chi_{\Gamma_{0}}\right) .
\end{aligned}
$$

Take the limit as $\Gamma_{0}$ increases:

$$
f(u)=S^{* *}(\alpha)\left(\chi_{\Gamma}\right)=\alpha\left(S^{*}\left(\chi_{\Gamma}\right)\right)=\alpha(f) .
$$

Thus $\alpha=u \in \mathfrak{X}$. This shows $\mathfrak{X} \prec l_{1}$.

The referee suggested that I close the paper with some questions. I do not know the answers to the following, although I have not worked on all of them. 
a. Is the converse of Theorem 13 true? I think this is the most interesting question listed here.

b. If $\mathfrak{X} \prec l_{1}$, does it follows that $L_{1}([0,1], \mathfrak{X}) \prec l_{1}$ ? This is suggested by Proposition 15, which shows that if $l_{1}(\Gamma) \prec l_{1}$ and $\mathfrak{X} \prec l_{1}$ then $l_{1}(\Gamma, \mathfrak{X}) \prec l_{1}$. More generally, one could ask: If $\mathfrak{X} \prec l_{1}$ and $\mathfrak{Y} \prec l_{1}$, does it follows that $\mathfrak{X} \hat{\otimes} \mathfrak{Y} \prec l_{1}$ ? (Note that some related conjectures are refuted by the observation that $l_{2} \hat{\otimes} l_{2}$ is not reflextive.) This is similar to the old problem: If $\mathfrak{X}$ and $\mathfrak{Y}$ are weakly sequentially complete, does it follows that $\mathfrak{X} \hat{\otimes} \mathfrak{Y}$ is weakly sequentially complete? (Recently refuted by Pisier.)

c. Is there a largest PIP. space? (Refer to Proposition 8.) Is there a space $\mathfrak{X}_{0}$ such that a Banach space $\mathfrak{X}$ has the PIP. if and only if $\mathfrak{X} \prec \mathfrak{X}_{0}$ ? If $\mu$ is a fixed measure, is there a space $\mathfrak{X}_{\mu}$ such that $\mathfrak{X}$ has the $\mu$-PIP. if and only if $\mathfrak{X} \prec \mathfrak{X}_{\mu}$ ?

d. Similarly, is there a largest weakly sequentially complete space? (Refer to Proposition 9.) Is there a largest separable weakly sequentially complete space?

e. Is there a third-smallest equivalence class? (Compare with Proposition 2.) Does the collection of all equivalence classes greater than $l_{1}$ have a least element? a minimal element?

f. Describe the poset of equivalence classes of separable Banach spaces. How many classes are there? What is the largest cardinality of a chain? of an antichain? Is there an infinite decreasing sequence?

Note added in proof. M. Talagrand has told me that he has constructed a weakly sequentially complete space that fails the condition on Borel measurable functionals mentioned after Proposition 9.

\section{REFERENCES}

1. J. Bourgain and F. Delbaen, A special class of $\mathcal{L}_{\infty}$ spaces, Acta Math., 145 (1980), $155-176$.

2. H. H. Corson, The weak topology of a Banach space, Trans. Amer. Math. Soc., 101 (1961), 1-15.

3. J. Diestel and J. J. Uhl, Vector Measures, Mathematical Surveys 15, Amer. Math. Soc., 1977.

4. N. Dunford and J. T. Schwartz, Linear operators, Part I, Interscience, 1957.

5. G. A. Edgar, Measurbility in a Banach space, Indiana Univ. Math. J., 26 (1977), 663-677.

6. $559-579$.

, Measurability in a Banach space, II, Indiana Univ. Math. J., 28 (1979),

7. A long James space, In: Measure Theory, Oberwolfach 1979, edited by D. Kolzow, Lecture Notes in Mathematics 794, Springer-Verlag, 1980.

8. D. H. Fremlin and M. Talagrand, $A$ decomposition theorem for additive set-functions, with applications to Pettis integrals and ergodic means, Math. Z., 168 (1979), 117-142. 
9. G. Godefroy and M. Talagrand, Classes d'espaces de Banach à prédual unique, C. R. Acad. Sci. Paris, 292 (1981), 323-325.

10. N. Kalton and A. Wilansky, Tauberian operators on Banach spaces, Proc. Amer. Math. Soc., 57 (1976), 251-255.

11. J. Lindenstrauss and L. Tzafriri, Classical Banach Spaces I, Ergebnisse der Mathematik und ihrer Grenzgebiete 92, Springer-Verlag, 1977.

12. H. P. Rosenthal and J. Bourgain, Applications of the theory of semi-embeddings to Banach space theory, preprint.

13. M. Talagrand, Certaines formes linéaires pathologiques sur un espace de Banach dual, Israel J. Math., 35 (1980), 171-176.

Received December 16, 1981 and in revised form March 8, 1982. Supported in part by N. S. F. Grant MCS 8003078. AMS 1980 subject classification. 46B20.

The Ohio State University

Columbus, OH 43210 


\section{PACIFIC JOURNAL OF MATHEMATICS EDITORS}

Donald BABBITT (Managing Editor)

University of California

Los Angeles, CA 90024

Hugo Rossi

University of Utah

Salt Lake City, UT 84112

C. C. Moore and Arthur Ogus

University of California

Berkeley, CA 94720
J. DugundiI

Department of Mathematics

University of Southern California

Los Angeles, CA 90089-1113

R. Finn and H. SAMELSON

Stanford University

Stanford, CA 94305

ASSOCIATE EDITORS
R. ARENS
E. F. BECKENBACH
B. H. NeUmanN
F. WOLF
K. YosHIDA (1906-1982)

\section{SUPPORTING INSTITUTIONS}

UNIVERSITY OF ARIZONA

UNIVERSITY OF BRITISH COLUMBIA

CALIFORNIA INSTITUTE OF TECHNOLOGY

UNIVERSITY OF CALIFORNIA

MONTANA STATE UNIVERSITY

UNIVERSITY OF NEVADA, RENO

NEW MEXICO STATE UNIVERSITY

OREGON STATE UNIVERSITY
UNIVERSITY OF OREGON

UNIVERSITY OF SOUTHERN CALIFORNIA

STANFORD UNIVERSITY

UNIVERSITY OF HAWAII

UNIVERSITY OF TOKYO

UNIVERSITY OF UTAH

WASHINGTON STATE UNIVERSITY

UNIVERSITY OF WASHINGTON 


\section{Pacific Journal of Mathematics}

\section{Vol. 108, No. $1 \quad$ March, 1983}

Waleed A. Al-Salam and A. Verma, $q$-Konhauser polynomials $\ldots \ldots \ldots \ldots 1$

Alfred David Andrew, The Banach space JT is primary $\ldots \ldots \ldots \ldots \ldots . . .6$

Thomas E. Bengtson, Bessel functions on $P_{n} \ldots \ldots \ldots \ldots \ldots \ldots$

Joaquim Bruna Floris and Francesc Tugores, Free interpolation for

holomorphic functions regular to the boundary $\ldots \ldots \ldots \ldots \ldots \ldots \ldots \ldots$

Peter Dierolf and Susanne Dierolf, Topological properties of the dual pair

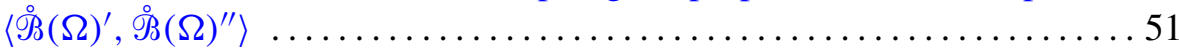

Gerald Arthur Edgar, An ordering for the Banach spaces $\ldots \ldots \ldots \ldots \ldots 83$

Basil Gordon, A proof of the Bender-Knuth conjecture . . . . . . . . . . . . . 99

Harold T. Hodes, A minimal upper bound on a sequence of Turing degrees

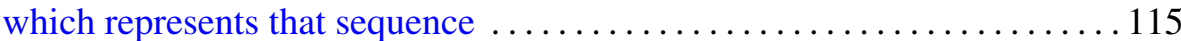

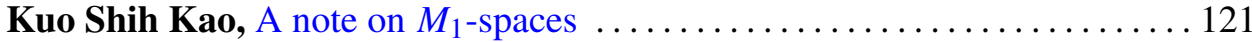

Frank Kost, Topological extensions of product spaces ................ 129

Eva Lowen-Colebunders, On the convergence of closed and compact

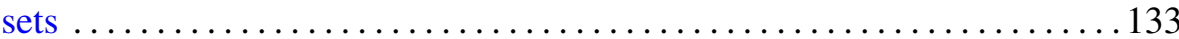

Doron Lubinsky, Divergence of complex rational approximations . . . . . . 141

Warren May and Elias Hanna Toubassi, Endomorphisms of rank one

mixed modules over discrete valuation rings $\ldots \ldots \ldots \ldots \ldots \ldots \ldots \ldots \ldots$

Richard Patrick Morton, The quadratic number fields with cyclic

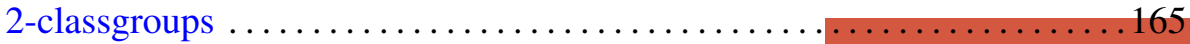

Roderic Murufas, Rank of positive matrix measures . . . . . . . . . . . . 177

Helga Schirmer, Fixed point sets of homotopies . . . . . . . . . . . . . 191

E. Taflin, Analytic linearization of the Korteweg-de Vries equation ........ 203

James Thomas Vance, Jr., $L^{p}$-boundedness of the multiple Hilbert

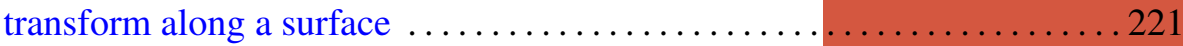

Hiroshi Yamaguchi, A property of some Fourier-Stieltjes transforms . . . . . 243 